\title{
Magnolol restores the activity of meropenem against NDM-1-producing Escherichia coli by inhibiting the activity of metallo-beta-lactamase
}

\author{
Shui Liu', Yonglin Zhou', Xiaodi Niu², Tingting Wang ${ }^{1}$, Jiyun $\mathrm{Li}^{3}$, Zhongjie Liư ${ }^{3}$, Jianfeng Wang ${ }^{1}$, Shusheng Tang ${ }^{3}$, \\ Yang Wang ${ }^{3}$ and Xuming Deng ${ }^{1}$
}

\begin{abstract}
The emergence of plasmid-mediated New Delhi metallo- $\beta$-lactamase-1 (NDM-1) in carbapenem-resistant Gramnegative pathogens is an increasing clinical threat. Here we report the discovery of an NDM-1 inhibitor, magnolol, through enzyme inhibition screening. We showed that magnolol significantly inhibited NDM enzyme activity $\left(I_{50}=\right.$ $6.47 \mu \mathrm{g} / \mathrm{mL}$ ), and it restored the activity of meropenem against Escherichia coli ZC-YN3, an NDM-1-producing E. coli isolate, in in vitro antibacterial activity assays. Magnolol lacked direct antibacterial activity, but compared with meropenem alone, it reduced the MICs of meropenem against E. coli ZC-YN3 by 4-fold and killed almost all the bacteria within $3 \mathrm{~h}$. Molecular modeling and a mutational analysis demonstrated that magnolol binds directly to the catalytic pocket (residues 110 to 200) of NDM-1, thereby blocking the binding of the substrate to NDM-1 and leading to its inactivation. Our results demonstrate that the combination of magnolol and meropenem may have the potential to treat infections caused by NDM-1-positive, carbapenem-resistant Gram-negative pathogens.
\end{abstract}

\section{Introduction}

The emergence and dissemination of multidrug-resistant pathogens, especially Gram-negative bacteria that encode extended-spectrum $\beta$-lactamases and are resistant to almost all currently available $\beta$-lactam antibiotics, is a worldwide public health problem ${ }^{1,2}$. Potent carbapenems, such as meropenem and imipenem, were once regarded as the last line of defense against multidrug-resistant Gramnegative bacteria ${ }^{3}$ as they are relatively stable in the presence of most bacterial $\beta$-lactamases, including extendedspectrum $\beta$-lactamases ${ }^{4}$. However, the increasing use of

Correspondence: Yang Wang (wangyang@cau.edu.cn) or Xuming Deng (dengxm@jlu.edu.cn)

'Key Laboratory of Zoonosis, Ministry of Education, Institute of Zoonosis, College of Veterinary Medicine, Jilin University, Changchun, China

${ }^{2}$ Department of Food Quality and Safety, Jilin University, Changchun, China Full list of author information is available at the end of the article

These authors contributed equally: Shui Liu and Yonglin Zhou.

Edited by A Rufini carbapenems created a vicious cycle that gave rise to carbapenem-resistant Gram-negative pathogens ${ }^{5,}{ }^{6}$. New Delhi metallo- $\beta$-lactamase-1 (NDM-1), classified as an Ambler classB1 metallo- $\beta$-lactamase $(\mathrm{MBL})^{7}$ and first identified in Klebsiella pneumoniae ${ }^{8}$, is a carbapenemase that was spread globally among multi-drug resistant bacterial pathogens ${ }^{9}$. NDM-1 gives enteric bacteria the ability to inactivate almost all $\beta$-lactams, including carbapenems ${ }^{10}$, and this MBL is not inhibited by any existing $\beta$-lactamase inhibitors ${ }^{11-14}$. Therefore, the presence of NDM-1 and its closely related variants in Enterobacteriaceae is threatening to diminish the effectiveness of carbapenems ${ }^{15}$, forcing the World Health Organization to issue a global warning ${ }^{16}$.

MBLs use one or two zinc ions in their active site to activate a nucleophilic water molecule that cleaves the lactam ring $^{7}$. Although they were first identified half a century ago ${ }^{17}$, many MBLs, such as the Verona integron- 
A

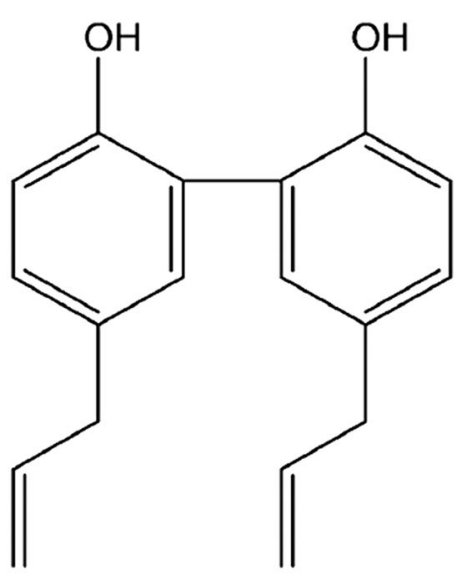

$\mathrm{C}$

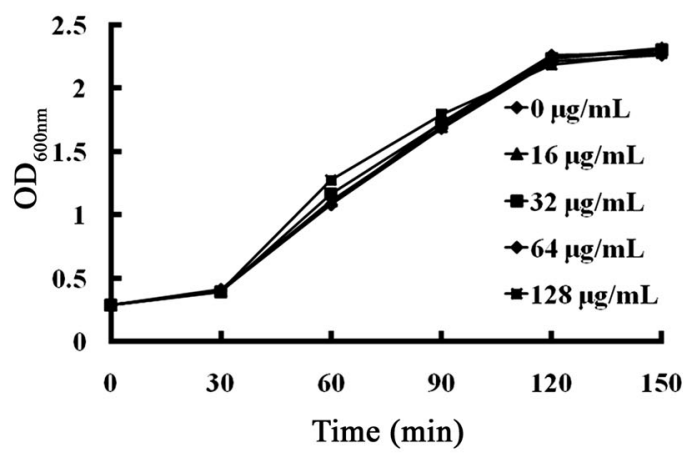

B

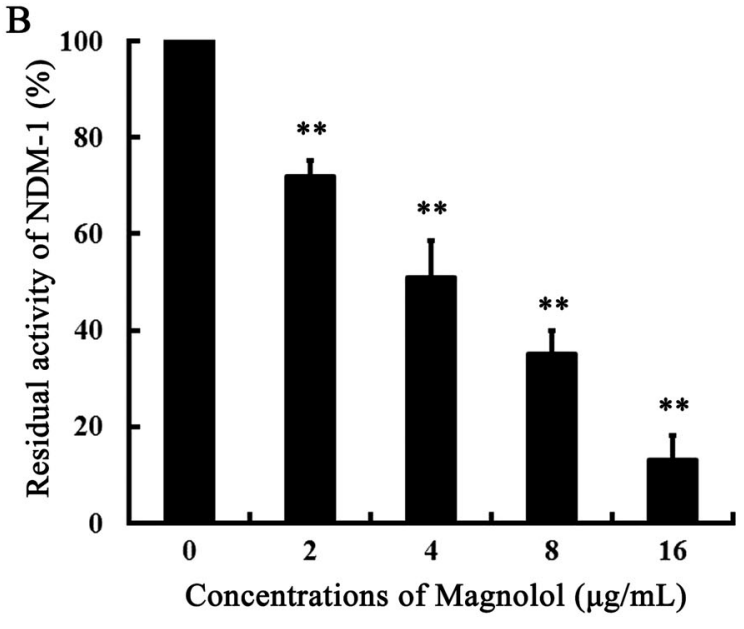

D

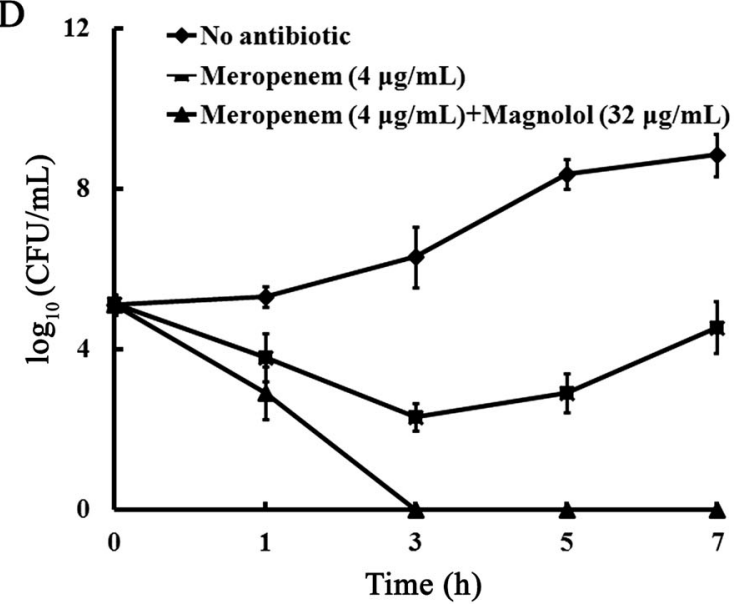

Fig. 1 Magnolol-mediated inhibition of E. coli ZC-YN3 in vitro. a Chemical structure of magnolol. b Magnolol inhibits NDM-1 activity. The values are the averages of three independent experiments. ${ }^{* *}$ indicates $P<0.01$. c Growth curves for $E$. coli ZC-YN3 cultured with magnolol. Representative data are one of three independent experiments. $\mathbf{d}$ Time-kill curves of compounds against $E$. coli ZC-YN3. The values are the averages of three independent experiments

encoded MBL (VIM) and imipenemase (IMP) variants, were only present in less pathogenic species, and genes encoding these MBLs were chromosomally located in most isolates. As a result, they have long been neglected in clinical settings ${ }^{17}$. In recent years, the $b a_{\mathrm{NDM}-1}$ gene was first identified in a transferable plasmid, and soon after, various horizontal gene transfer elements permitted NMD1 and other resistance genes to spread rapidly and globally $^{10}$. As bla NDM-1 $_{1}$ is one of the major driving forces for the rapid spread of carbapenem resistance that has resulted in a great crisis that threatens the use of $\beta$-lactam antibiotics to treat infections caused by NDM-1-producing pathogens, it is considered to be the main target for novel effective inhibitors ${ }^{18}$. Despite the high unmet medical necessity, few effective clinical inhibitors of NDM- 1 and other MBLs have been reported so $\mathrm{far}^{19,20}$.

Here we identified a potent inhibitor of the NDM-1 enzyme, magnolol (Fig. 1a), a natural compound isolated from the bark of magnolia (Magnolia officinalis Rehder and EH Wilson) trees, using a purified NDM-1 protein and the $\beta$-lactamase substrate nitrocefin screening approach. By binding to the active site of NDM-1, magnolol effectively inhibited the biological activity of NDM-1 and successfully rescued the effectiveness of meropenem in vitro against NDM-1-expressing E. coli. The combination of magnolol and meropenem may have the potential to treat infections caused by NDM-1-positive, carbapenem-resistant Gram-negative pathogens.

\section{Results}

Magnolol-mediated inhibition of Escherichia coli ZC-YN3 in vitro

Enzyme inhibition assays using purified recombinant NDM-1 enzymes demonstrated that among 75 natural compounds, only magnolol had a significant impact on MBL enzyme activity in vitro (Fig. 1b). NDM-1 activity 
(27.92\%) was inhibited significantly by adding $2 \mu \mathrm{g} / \mathrm{mL}$ magnolol, and minimal activity (13.24\%) was detected in the groups treated with $16 \mu \mathrm{g} / \mathrm{mL}$ magnolol $\left(\mathrm{IC}_{50}=6.47\right.$ $\mu \mathrm{g} / \mathrm{mL}$ ). Moreover, further adding excessive zinc (100 mM

Table 1 MIC $(\mu \mathrm{g} / \mathrm{mL})$ of meropenem against $E$. coli isolates

\begin{tabular}{lll}
\hline Strains & Meropenem & Combination \\
\hline E. coli ZC-YN3 (NDM-1) & 16 & $4(\mathbf{4})$ \\
E. coli ZC-YN5 (NDM-5) & 32 & $8(\mathbf{4})$ \\
E. coli ZC-YN7 (NDM-9) & 64 & $16(\mathbf{4})$ \\
\hline
\end{tabular}

All MICs were determined in triplicate. Magnolol in combination with meropenem was tested at a final concentration of $32 \mu \mathrm{g} / \mathrm{mL}$. The fold change is indicated in bold
$\mathrm{ZnCl}_{2}$ ) to the mixture of magnolol and NDM- 1 for $12 \mathrm{~h}$ did not restore the NDM-1 activity (data not shown), indicating that the magnolol-mediated inhibition of NDM-1 may not consistent with a metal-depletion mechanism $^{21}$.

Subsequently, magnolol was subjected to antimicrobial testing using $E$. coli isolates in the absence of meropenem. Since Class B1 MBLs generally exhibit significant structural similarity with the ions and disposition of the catalytic residues, inhibitors often work on all $\mathrm{MBLs}^{22}$. As shown in Table 1, meropenem plus magnolol reduced the MICs of meropenem against the E. coli ZC-YN3 (producing NDM-1), ZC-YN5 (producing NDM-5, two amino acids differ from NDM-1) and ZC-YN7 (producing NDM9, a single amino acid substitution from NDM-1) by 4-fold compared with meropenem alone. Notably, the fractional inhibitory concentration value of this combination was

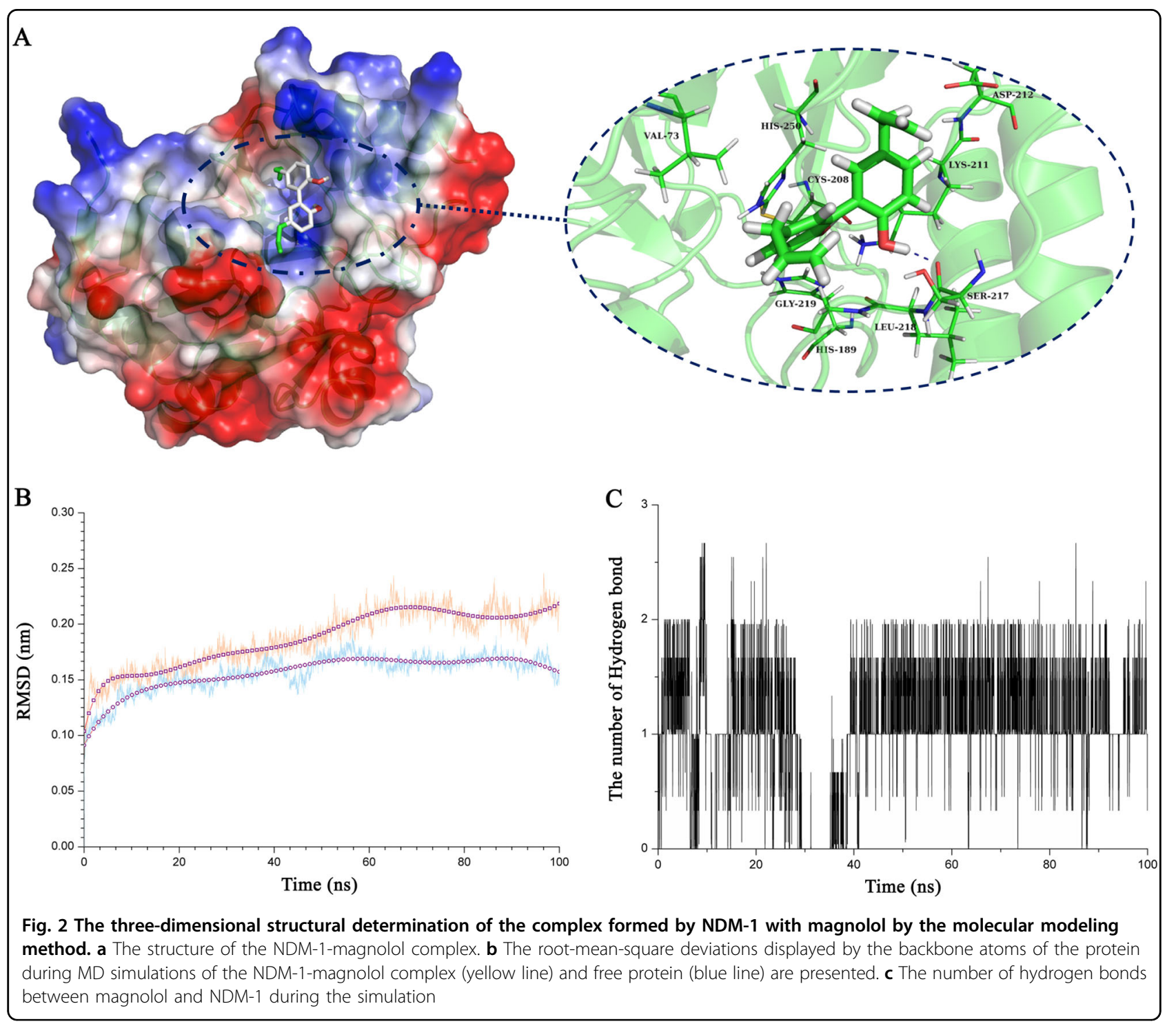




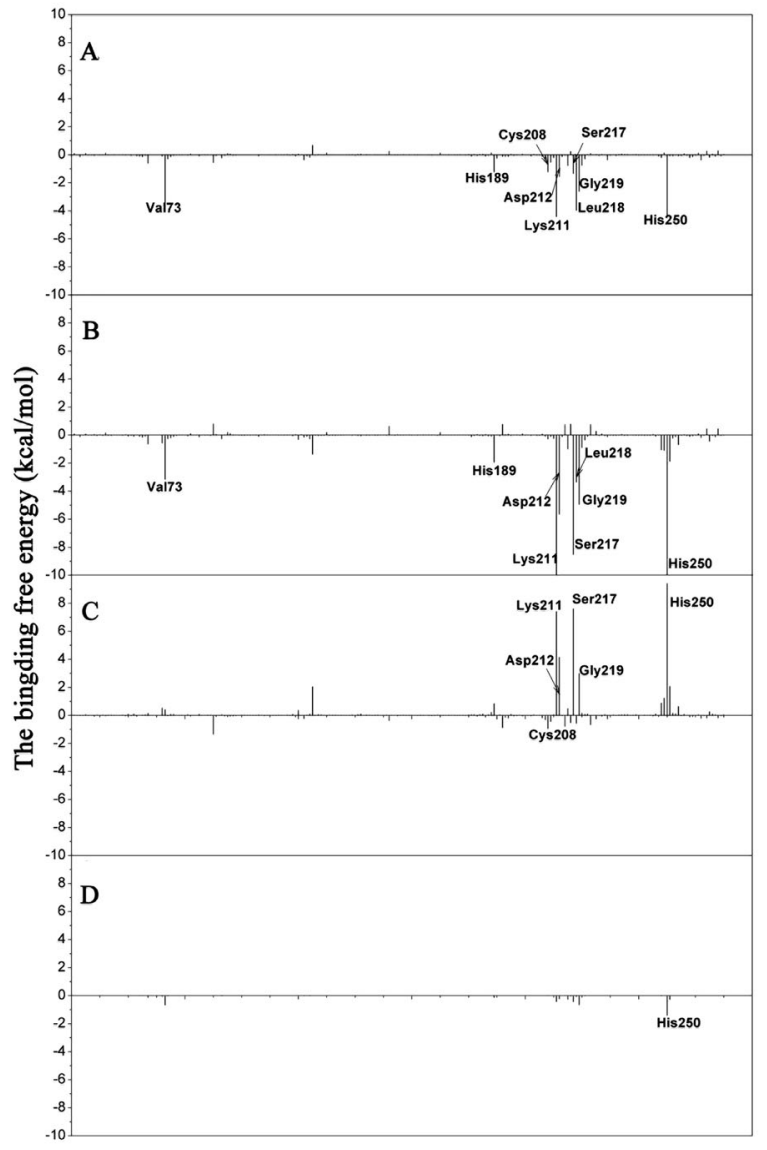

E
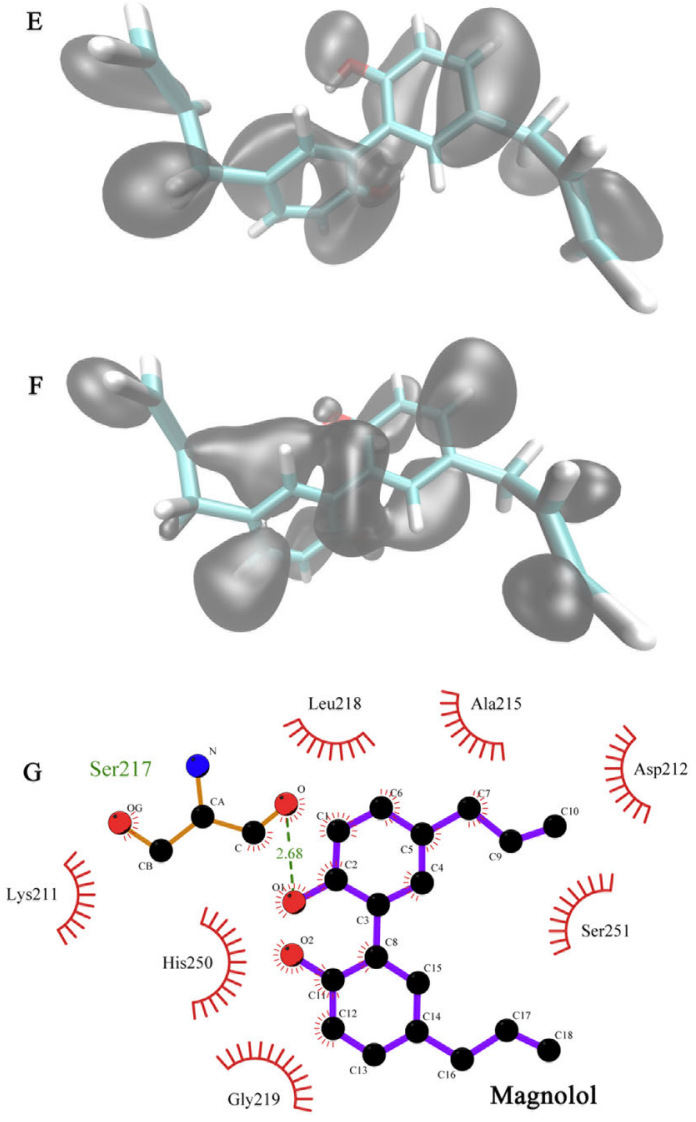

Fig. 3 The predicted interaction mechanism between magnolol and NDM-1. Decomposition of the binding energy on a per-residue basis in the WT-NDM-1-magnolol complex. The histogram chart shows the total (a), van der Waals (b), electrostatic (c), and solvation (d) contributions for the complexes. The highest occupied molecular orbital (e) and lowest unoccupied molecular orbital (f) of magnolol. $\mathbf{g}$ Interactions between magnolol and the residues of the binding sites in NDM-1 are shown using a two-dimensional diagram by Ligplus

0.281 , suggesting that these treatments showed synergistic activity. Meanwhile, magnolol alone exhibited no efficient antibacterial effect $(\mathrm{MIC}>1,024 \mu \mathrm{g} / \mathrm{mL})$, and it also had no influence on the growth of NDM-1-producing E. coli ZC-YN3 (Fig. 1c).

In agreement with the synergy mentioned above, timekill curves demonstrated that the combination of magnolol plus meropenem exerted killing effects on $E$. coli $\mathrm{ZC}-\mathrm{YN} 3$, thoroughly killing the bacteria by $3 \mathrm{~h}$ after coincubation (Fig. 1d). Our results indicate that magnolol restored the activity of meropenem against $E$. coli ZCYN3 in vitro.

\section{Molecular dynamics (MD) simulation for the NDM-1- magnolol complex}

Using a computational biology method, we explored the potential binding mode of magnolol to the active site of NDM-1 (Fig. 2a). It is obvious that magnolol can bind to NDM-1 via hydrogen bonding and hydrophobic interactions. During the time course of the simulation, magnolol localized to the catalytic pocket of NDM-1 (residues 110 to 200). In detail, the binding model of magnolol to NDM-1 revealed that the side chain of magnolol can form one hydrogen bond with Ser217. The complex reached equilibrium at $100 \mathrm{~ns}$ based on ananalysis of the rootmean-square deviations of the backbone $C \alpha$ atoms (Fig. 2b). Moreover, the number of hydrogen bonds was calculated during the simulation. The number of hydrogen bonds fluctuated between one and two during the simulation, further confirming that there is one hydrogen bond between magnolol and NDM-1 (Fig. 2c).

To explore the energy contributions from the residues of the binding sites in the NDM-1-magnolol complex, the energy decomposition was calculated for the NDM-1magnolol complex. Five residues, Val73, Lys211, Leu218, Gly219, and His250, made a strong total binding energy contribution, with a $\Delta E_{\text {total }}$ of $\leq-2.0 \mathrm{kcal} / \mathrm{mol}$ (Fig. 3a). In addition, residues His189, Cys208, Asp212, and Ser217 also contributed appreciably to the total binding energy, with a $\Delta E_{\text {total }}$ of $\leq-1.0 \mathrm{kcal} / \mathrm{mol}$. These results suggest 
that these five residues (Val73, Lys211, Leu218, Gly219, and His250) are key residues for magnolol binding. As shown in Fig. 3b-d, most of the decomposed energy interaction originated from van der Waals interactions, apparently through hydrophobic interactions, while the electrostatic contribution appeared to have an unfavorable influence on these key residues during complex formation. The magnolol conformation was optimized with the B3LYP/6-311G" set with Gaussian 09 software. As shown in Fig. 3e, f, the highest occupied molecular orbital and the lowest unoccupied molecular orbital of magnolol indicated that the benzene ring is the active binding center of magnolol (Fig. 3g).

Among these above-mentioned five key residues, since the Val73 is located outside the catalytic pocket and irrelevant for the activity of NDM-1, and the Leu218 and His250 mutant may induce a conformation change in NDM-1 and further affect the binding ability of magnolol to other residues; therefore, we selected the Lys211Ala and Gly219Ala mutants to confirm these theoretical results. The total binding free energy for the NDM-1magnolol complex and their detailed energy contributions were calculated according to the molecular mechanics Poisson-Boltzmann surface area approach, and they are summarized in Table 2 . The binding free energy, $\Delta G_{\text {bind }}$, of the interaction between magnolol and wild-type (WT) NDM-1 was greater than that of the Lys211Ala and Gly219Ala mutants, which means that WT-NDM-1 has the strongest ability to bind magnolol. Via fluorescence spectroscopy quenching, we measured $\Delta G_{b i n d}$ and the number of binding sites between magnolol and the Lys211Ala and Gly219Ala mutants, and these results were highly consistent with those obtained by computational methods (Table 2), further conforming the information generated by the MD simulation of the NDM-1-magnolol complex, namely, that because of the binding of the inhibitor magnolol to the active site region (residues Val73, Lys211, Leu218, His189, Cys208, Asp212, Ser217, Gly219, and His250), the biological activity of NDM-1 was largely inhibited (Fig. 4a).

Table 2 The binding free energy $(\mathrm{kcal} / \mathrm{mol})$ of the WTNDM-1-magnolol, Lys211 Ala-magnolol, and Gly219Alamagnolol complexes based on computational methods and the values of the binding constants $\left(K_{A}\right)$ based on fluorescence spectroscopy quenching

\begin{tabular}{llll}
\hline & WT-NDM-1 & K211A & G219A \\
\hline The binding free energy & $-13.6 \pm 2.1$ & $-7.9 \pm 1.2$ & $-8.6 \pm 1.6$ \\
$K_{A}\left(1 \times 10^{4}\right) \mathrm{L} / \mathrm{mol}$ & $8.6 \pm 1.7$ & $4.8 \pm 1.1$ & $5.5 \pm 1.3$ \\
\hline
\end{tabular}

All tests were determined in triplicate

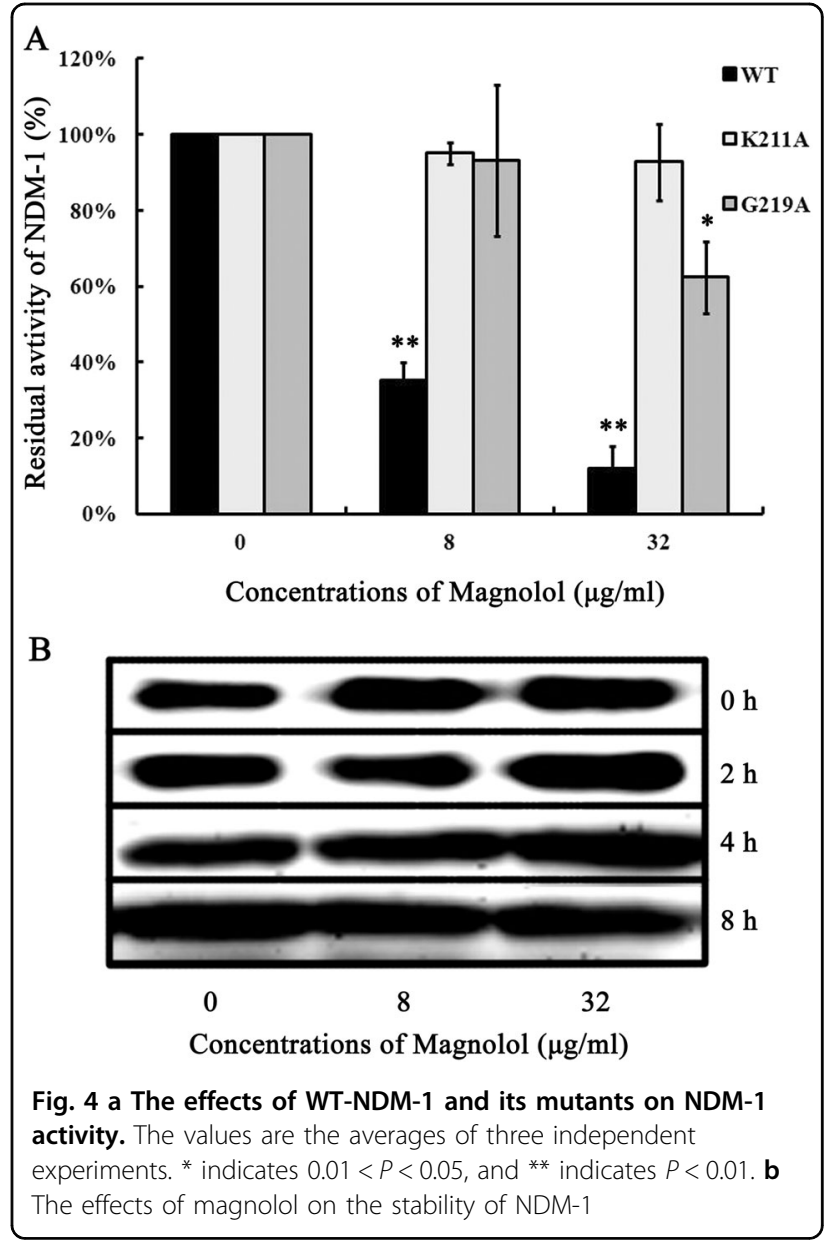

\section{Magnolol has no effect on the stability of NDM-1}

As shown in Fig. 4b, the stability of NDM-1 was not affected after $0,2,4$, or $8 \mathrm{~h}$ of incubation with different concentrations of magnolol. These data indicate that magnolol has no impact on the stability of NDM-1.

\section{Discussion}

MBLs are the major targets for developing efficient inhibitors against carbapenem-resistant Enterobacteriacea $^{18}$. Through unremitting efforts, various types of MBL inhibitors with different mechanisms have been described. In 2014, King et al. identified a fungal natural product, aspergillomarasmine A (AMA), which is as an effective inhibitor of NDM-1 and VIM-2, through a cellbased screening approach ${ }^{21}$. AMA overcame the resistance mediated by these two MBLs by affecting NDM-1/ VIM-2-bound zinc, and it fully restored the antibacterial activity of meropenem. In a mouse model, AMA was shown to be a potential therapeutic agent, but one of the major challenges to its clinical application is the difficulty associated with its chemical synthesis. Fortunately, Liao 
et al. reported the first total synthesis and stereochemical configuration reassignment of AMA that is amenable to the efficient preparation of $\mathrm{AMA}^{20}$. In addition, research by Chiou et al. demonstrated that ebselen, which is an anti-oxidant drug already safely used in human studies, might be a promising inhibitor of NDM-1 by targeting the Cys residue at the active site. However, its anti-oxidant activity and toxicity might limit its potential ${ }^{23}$. Klingler et al. tested 11 approved drugs containing a thiol moiety, and they found four approved drugs (captopril, thiorphan, dimercaprol, and tiopronin) possessed inhibitory activity for NDM-1, VIM-1, and IMP-7. However, the concentrations required to restore their antibacterial activity could not be reached in pathogens ${ }^{24}$. For practical and technical reasons, to date, there are few inhibitors with established potential for clinical application. Thus, the development of MBL inhibitors to restore the activity of $\beta$-lactam antibiotics is of great necessity.

Natural compounds have played an important role in the discovery of antibiotics. Magnolol, an abundant natural compound isolated from $M$. officinalis, has been used widely in traditional Chinese medicine ${ }^{25}$. Here we showed that magnolol inhibited the activity of NDM-1, which was confirmed by in vitro experiments. Compared with the above inhibitors, magnolol has the advantages of abundant sources and easy preparation ${ }^{26,27}$. The high hydrophobicity and low solubility of magnolol may be the major obstacles to its bioavailability and clinical efficacy ${ }^{28,} 29$. However, we revealed that the concentrations required to restore antimicrobial activity could be achieved in bacteria. Moreover, magnolol inhibited NDM-1 activity without impacting NDM-bound zinc, which differs from the metal-depletion mechanisms of AMA. The toxicity associated with cross reactivity with human metalloenzymes is a major challenge for the development of MBL inhibitors ${ }^{21}$. Known chelators, such as EDTA, have been greatly restricted in clinical use because of these side effects. For instance, the median lethal dose of EDTA was calculated to be $28.5 \mathrm{mg} / \mathrm{kg}$ when administered intravenously in mice ${ }^{30}$. Magnolol has little toxicity in vivo because of the differences in its mode of action compared with those of other metal-ion-chelating agents. Animal studies demonstrated that magnolol showed no clinical signs of toxicity in mice and rats ${ }^{31,32}$. Notably, magnolol has very low toxicity in dogs (no mortality at $1 \mathrm{~g} / \mathrm{kg}$ when administered intravenously to dogs) ${ }^{33}$. Thus, these data indicate that magnolol may be a potentially safe inhibitor of NDM-1.

In conclusion, our data demonstrate that magnolol inhibited $\beta$-lactamase enzymatic activity by binding to the active site of NDM-1, and it restored the activity of meropenem against NDM-1-positive E. coli isolates. In vitro, synergistic activity was observed with the combination of magnolol plus meropenem. Taken together, these results identified a potential clinically efficacious dose using in vitro, which will contribute to the future development of an effective NDM-1 inhibitor.

\section{Materials and methods \\ Bacterial strains and chemicals}

The NDM-1-producing E. coli isolates was originated from our previous study ${ }^{34}$. Magnolol ( $\geq 98 \%$ pure) and meropenem ( $\geq 87 \%$ pure) were purchased from the National Institutes for Food and Drug Control (Beijing, China). Stock solutions of magnolol were prepared in dimethyl sulfoxide (DMSO, Sigma-Aldrich, St. Louis, MO, USA). Meropenem was dissolved in sterile water.

\section{Plasmid construction and protein purification}

To produce recombinant NDM-1 in E. coli, a pET28aNDM-1 plasmid with the restriction sites BamHI and Xhol was constructed. A $b l a_{\mathrm{NDM}-1}$ gene without the signal peptide was amplified from strain E. coli ZC-YN3 with the primers NDM-1-F/NDM-1-R (Table S1). This vector encodes the intact NDM-1 sequence fused to an aminoterminal histidine tag. The Lys211Ala and Gly219Ala mutations of NDM-1 were introduced into pET28aNDM-1 using the Quick Change Site-directed Mutagenesis Kit (Stratagene, San Diego, CA, USA) with the primers K211A-F/K211A-R and G219A-F/G219A-R, respectively (Table S1). All constructed strains were verified by PCR and sequencing. Protein expression was performed according to Liao et $\mathrm{al}^{20}$.

\section{Enzyme inhibition assays}

The assay was conducted according to Liao et al.'s method $^{20}$ with minor modifications. To find NDM-1 inhibitors, we selected 75 natural compounds as screening compounds (Table S2). Assays were read in 96-well plates at an absorbance of $492 \mathrm{~nm}$ using a microplate reader (Tecan Austria GmbH, Grödig, Austria) at room temperature. Positive controls were performed in the presence of enzyme and in the absence of inhibitors, whereas negative controls were performed in the absence of enzyme. Residual activity $=\mathrm{A}-\mathrm{A}_{0} / \mathrm{A}_{100}-\mathrm{A}_{0} \times 100 \%$, where A represents the absorbance of inhibitor groups at $492 \mathrm{~nm}$, and $\mathrm{A}_{0}$ and $\mathrm{A}_{100}$ represent $0 \%$ and $100 \%$ activity as determined in the negative controls and positive controls, respectively.

\section{Antibacterial activity assays in vitro}

MICs of magnolol, meropenem, and combinations of magnolol plus meropenem against $E$. coli isolates were determined using the broth microdilution method following the guidelines of the Clinical and Laboratory Standards Institute. The combinations were evaluated by calculating the fractional inhibitory concentration (FIC) index values. To evaluate the effect of magnolol on the 
growth of the tested strains, a growth curve assay was performed. Specifically, E. coli ZC-YN3 was cultured in Luria-Bertani medium at $37^{\circ} \mathrm{C}$ with shaking $(180 \mathrm{rpm})$ to an optical density at $600 \mathrm{~nm}$ of 0.3 and then aliquoted into five 50-mL conical flasks. Magnolol (or the DMSO control) was added to the five cultures at $0,16,32,64$, and $128 \mu \mathrm{g} / \mathrm{mL}$. The bacteria were cultured at $37^{\circ} \mathrm{C}$ with constant shaking, and cell growth was estimated by measuring the $\mathrm{OD}_{600}$ every $30 \mathrm{~min}$. In addition, the potential bactericidal effect of magnolol combined with meropenem was evaluated by time-killing assays ${ }^{35}$.

\section{Molecular modeling}

The initial structure of NDM-1 was obtained from the three-dimensional X-ray structure (PDB code: 4EXS). To obtain the starting structure of the magnolol/NDM-1 complex for the MD simulation, a standard docking procedure for a rigid protein and a flexible ligand was performed with AutoDock $4^{36,37}$. Subsequently, the MD simulation of the complex was performed. The processes of the computational biology method have been described in detail in previous reports ${ }^{38,} 39$.

\section{Determination of the binding affinity of magnolol to mutant NDM-1 proteins}

The fluorescence-quenching method was used to measure the binding constants $\left(K_{A}\right)$ of magnolol with the NDM-1 mutants (Lys211Ala and Gly219Ala). A 280-nm excitation wavelength with a 5-nm bandpass and a 345$\mathrm{nm}$ emission wavelength with a $10-\mathrm{nm}$ bandpass were used for the measurements. Details of the measurements have been described previously ${ }^{40,41}$.

\section{NDM-1 stability assays}

For NDM-1 stability assays, purified NDM-1 was incubated without magnolol or with 8 and $32 \mu \mathrm{g} / \mathrm{mL}$ magnolol for $0,2,4$, and $8 \mathrm{~h}$ at $37^{\circ} \mathrm{C}$. Western blotting was performed to investigate the stability of NDM-1 treated with magnolol. Anti-histidine-tag antibodies (1:4,000 dilution, Proteintech Group, Inc., Rosemont, IL, USA) and horseradish peroxidase-conjugated goat antimouse antibodies (1:2,000 dilution, Proteintech Group, Inc.) were used as the primary and secondary antibodies, respectively.

\section{Statistical analysis}

Data are presented as the mean \pm standard deviation from three independent experiments, and they were analyzed using SPSS Statistics for Windows, version 19.0 (IBM Corp. Armonk, NY, USA). Significant differences were determined using an independent Student's $t$-test as indicated. Differences were considered statistically significant when $P$ values were less than 0.05 .

\section{Acknowledgements}

This work was supported by the National Key Technology R\&D Program (No. 2016YFD05013) and the National Natural Science Foundation of China (31422055 and 81661138002).

\section{Author details}

${ }^{1}$ Key Laboratory of Zoonosis, Ministry of Education, Institute of Zoonosis, College of Veterinary Medicine, Jilin University, Changchun, China.

${ }^{2}$ Department of Food Quality and Safety, Jilin University, Changchun, China. ${ }^{3}$ College of Veterinary Medicine, China Agricultural University, Beijing, China

\section{Conflict of interest}

The authors declare that they have no conflict of interest.

\section{Publisher's note}

Springer Nature remains neutral with regard to jurisdictional claims in published maps and institutional affiliations.

Supplementary Information accompanies this paper at https://doi.org/ 10.1038/s41420-018-0029-6

Received: 22 November 2017 Accepted: 22 December 2017 Published online: 20 February 2018

\section{References}

1. Spellberg, B. et al. The epidemic of antibiotic-resistant infections: a call to action for the medical community from the Infectious Diseases Society of America. Clin. Infect. Dis. 46, 155-164 (2008).

2. Brown, E. D. \& Wright, G. D. Antibacterial drug discovery in the resistance era. Nature 529, 336-343 (2016).

3. Alm, R. A., Johnstone, M. R. \& Lahiri, S. D. Characterization of Escherichia coli NDM isolates with decreased susceptibility to aztreonam/avibactam: role of a novel insertion in PBP3. J. Antimicrob. Chemother. 70, 1420-1428 (2015).

4. Schneider, K. D., Karpen, M. E., Bonomo, R. A., Leonard, D. A. \& Powers, R. A. The 1.4 A crystal structure of the class D beta-lactamase OXA-1 complexed with doripenem. Biochemistry 48, 11840-11847 (2009).

5. Karaiskos, I. \& Giamarellou, H. Multidrug-resistant and extensively drug-resistant Gram-negative pathogens: current and emerging therapeutic approaches. Expert Opin. Pharmacother. 15, 1351-1370 (2014).

6. Livermore, D. M. Has the era of untreatable infections arrived? J. Antimicrob. Chemother. 64(Suppl 1), i29-i36 (2009).

7. Bush, K. \& Jacoby, G. A. Updated functional classification of beta-lactamases Antimicrob. Agents Chemother. 54, 969-976 (2010).

8. Yong, D. et al. Characterization of a new metallo-beta-lactamase gene, bla (NDM-1), and a novel erythromycin esterase gene carried on a unique genetic structure in Klebsiella pneumoniae sequence type 14 from India. Antimicrob. Agents Chemother. 53, 5046-5054 (2009).

9. Berrazeg, M. et al. New Delhi Metallo-beta-lactamase around the world: an eReview using Google Maps. Eur. Surveill. 19, 20 (2014).

10. Kumarasamy, K. K. et al. Emergence of a new antibiotic resistance mechanism in India, Pakistan, and the UK: a molecular, biological, and epidemiological study. Lancet Infect. Dis. 10, 597-602 (2010).

11. Struelens M. J., Monnet D. L., Magiorakos A. P., Santos O'Connor F., Giesecke J. European NDMSP. New Delhi metallo-beta-lactamase 1-producing Enterobacteriaceae: emergence and response in Europe. Eur. Surveill. 15, 9-16 (2010).

12. Nordmann, P., Poirel, L., Walsh, T. R. \& Livermore, D. M. The emerging NDM carbapenemases. Trends Microbiol. 19, 588-595 (2011).

13. Cornaglia, G., Giamarellou, H. \& Rossolini, G. M. Metallo-beta-lactamases: a last frontier for beta-lactams? Lancet Infect. Dis. 11, 381-393 (2011).

14. Nordmann, P., Poirel, L., Toleman, M. A. \& Walsh, T. R. Does broad-spectrum beta-lactam resistance due to NDM-1 herald the end of the antibiotic era for treatment of infections caused by Gram-negative bacteria? J. Antimicrob. Chemother. 66, 689-692 (2011).

15. Walsh, T. R. Emerging carbapenemases: a global perspective. Int. J. Antimicrob. Agents 36(Suppl 3), S8-S14 (2010). 
16. Hsu, L. Y. \& Koh, T. H. World Health Day 2011: combating antimicrobial resistance. Singap. Med. J. 52, 230-231 (2011).

17. Ellar, D. J. \& Lundgren, D. G. Fine structure of sporulation in Bacillus cereus grown in a chemically defined medium. J. Bacteriol. 92, 1748-1764 (1966).

18. Drawz, S. M. \& Bonomo, R. A. Three decades of beta-lactamase inhibitors. Clin. Microbiol. Rev. 23, 160-201 (2010).

19. Albu, S. A. et al. Total synthesis of Aspergillomarasmine A and related compounds: a sulfamidate approach enables exploration of structure-activity relationships. Angew. Chem. 55, 13259-13262 (2016).

20. Liao, D. et al. Total synthesis and structural reassignment of Aspergillomarasmine A. Angew. Chem. 55, 4291-4295 (2016).

21. King, A. M. et al. Aspergillomarasmine A overcomes metallo-beta-lactamase antibiotic resistance. Nature 510, 503-506 (2014)

22. King, D. T., Worrall, L. J., Gruninger, R. \& Strynadka, N. C. New Delhi metallobeta-lactamase: structural insights into beta-lactam recognition and inhibition. J. Am. Chem. Soc. 134, 11362-11365 (2012).

23. Chiou, J. et al. Ebselen as a potent covalent inhibitor of New Delhi metallobeta-lactamase (NDM-1). Chem. Commun. 51, 9543-9546 (2015).

24. Klingler, F. M. et al. Approved drugs containing thiols as inhibitors of metallobeta-lactamases: strategy to combat multidrug-resistant bacteria. J. Med. Chem. 58, 3626-3630 (2015).

25. Alexeev, M., Grosenbaugh, D. K., Mott, D. D. \& Fisher, J. L. The natural products magnolol and honokiol are positive allosteric modulators of both synaptic and extra-synaptic GABA(A) receptors. Neuropharmacology 62, 2507-2514 (2012).

26. Chen, L. et al. Rapid purification and scale-up of honokiol and magnolol using high-capacity high-speed counter-current chromatography. J. Chromatogr. A 1142, 115-122 (2007).

27. Lee, Y. J. et al. Therapeutic applications of compounds in the Magnolia family. Pharmacol. Ther. 130, 157-176 (2011).

28. Wang, Y. J., Chien, Y. C., Wu, C. H. \& Liu, D. M. Magnolol-loaded core-shell hydrogel nanoparticles: drug release, intracellular uptake, and controlled cytotoxicity for the inhibition of migration of vascular smooth muscle cells. Mol. Pharm. 8, 2339-2349 (2011).

29. He, S., Zhang, Z., Xu, F., Zhang, S. \& Lei, Z. Micronization of magnolia bark extract with enhanced dissolution behavior by rapid expansion of supercritical solution. Chem. Pharm. Bull. 58, 154-159 (2010).
30. Matsuura, A. et al. Pharmacological profiles of aspergillomarasmines as endothelin converting enzyme inhibitors. Jpn. J. Pharmacol. 63, 187-193 (1993).

31. Li, N. et al. Evaluation of the in vitro and in vivo genotoxicity of magnolia bark extract. Regul. Toxicol. Pharmacol. RTP 49, 154-159 (2007).

32. Liu, Z. et al. Evaluation of short-term and subchronic toxicity of magnolia bark extract in rats. Regul. Toxicol. Pharmacol. RTP 49, 160-171 (2007).

33. Poivre, M. \& Duez, P. Biological activity and toxicity of the Chinese herb Magnolia officinalis Rehder \& E. Wilson (Houpo) and its constituents. J. Zhejiang Univ. Sci. B 18, 194-214 (2017).

34. Wang, Y. et al. Comprehensive resistome analysis reveals the prevalence of NDM and MCR-1 in Chinese poultry production. Nat. Microbiol. 2, 16260 (2017).

35. Kang, W. et al. Effective antimicrobial activity of a peptide mutant Cbf-14-2 against penicillin-resistant bacteria based on its unnatural amino acids. Eur. J. Pharm. Sci. 105, 169-177 (2017).

36. Morris, G. M. et al. AutoDock4 and AutoDockTools4: automated docking with selective receptor flexibility. J. Comput. Chem. 30, 2785-2791 (2009).

37. Hu, R., Barbault, F., Maurel, F., Delamar, M. \& Zhang, R. Molecular dynamics simulations of 2-amino-6-arylsulphonylbenzonitriles analogues as HIV inhibitors: interaction modes and binding free energies. Chem. Biol. Drug Des. 76 518-526 (2010).

38. Dong, J. et al. Oroxylin A inhibits hemolysis via hindering the self-assembly of alpha-hemolysin heptameric transmembrane pore. PLoS Comput. Biol. 9, e1002869 (2013)

39. Niu, X. et al. Molecular insight into the inhibition mechanism of cyrtominetin to alpha-hemolysin by molecular dynamics simulation. Eur. J. Med. Chem. 62, 320-328 (2013).

40. Jurasekova, Z., Marconi, G., Sanchez-Cortes, S. \& Torreggiani, A. Spectroscopic and molecular modeling studies on the binding of the flavonoid luteolin and human serum albumin. Biopolymers 91, 917-927 (2009).

41. Bandyopadhyay, S., Valder, C. R., Huynh, H. G., Ren, H. \& Allison, W. S. The beta G156C substitution in the F1-ATPase from the thermophilic Bacillus PS3 affects catalytic site cooperativity by destabilizing the closed conformation of the catalytic site. Biochemistry 41, 14421-14429 (2002). 